\title{
Leitlinien für den Verbraucherschutz im Zusammenhang mit dem elektronischen Geschäftsverkehr
}


(c) OECD, 2000.

(C) Software: 1987-1996, Acrobat is a trademark of ADOBE.

All rights reserved. OECD grants you the right to use one copy of this Program for your personal use only. Unauthorised reproduction, lending, hiring, transmission or distribution of any data or software is prohibited. You must treat the Program and associated materials and any elements thereof like any other copyrighted material.

All requests should be made to:

Head of Publications Division

Public Affairs and Communication Directorate

2, rue André-Pascal, 75775 Paris

Cedex 16, France. 


\section{Leitlinien \\ für den Verbraucherschutz im Zusammenhang mit dem elektronischen Geschäftsverkehr}




\title{
ORGANISATION FÜR WIRTSCHAFTLICHE ZUSAMMENARBEIT UND ENTWICKLUNG
}

Gemäß Artikel 1 des am 14. Dezember 1960 in Paris unterzeichneten und am 30. September 1961 in Kraft getretenen Übereinkommens fördert die Organisation für wirtschaftliche Zusammenarbeit und Entwicklung (OECD) eine Politik, die darauf gerichtet ist:

- in den Mitgliedstaaten unter Wahrung der finanziellen Stabilität eine optimale Wirtschaftsentwicklung und Beschäftigung sowie einen steigenden Lebensstandard zu erreichen und dadurch zur Entwicklung der Weltwirtschaft beizutragen;

- in den Mitglied- und Nichtmitgliedstaaten, die in wirtschaftlicher Entwicklung begriffen sind, zu einem gesunden wirtschaftlichen Wachstum beizutragen, und

- im Einklang mit internationalen Verpflichtungen auf multilateraler und nichtdiskriminierender Grundlage zur Ausweitung des Welthandels beizutragen.

Die Gründungsmitglieder der OECD sind: Belgien, Dänemark, Deutschland, Frankreich, Griechenland, Irland, Island, Italien, Kanada, Luxemburg, Niederlande, Norwegen, Österreich, Portugal, Schweden, Schweiz, Spanien, Türkei, Vereinigtes Königreich und Vereinigte Staaten. Folgende Staaten wurden zu den nachstehend genannten Daten Mitglieder der OECD: Japan (28. April 1964), Finnland (28. Januar 1969), Australien (7. Juni 1971), Neuseeland (29. Mai 1973), Mexiko (18. Mai 1994), die Tschechische Republik (21. Dezember 1995), Ungarn (7. Mai 1996), Polen (22. November 1996) und Korea (12. Dezember 1996). Die Kommission der Europäischen Gemeinschaften nimmt an den Tätigkeiten der OECD teil (Artikel 13 des Übereinkommens über die OECD).

\author{
Originalfassungen veröffentlicht unter dem Titel : \\ GUIDELINES FOR CONSUMER PROTECTION IN THE CONTEXT OF ELECTRONIC COMMERCE \\ LIGNES DIRECTRICES RÉGISSANT LA PROTECTION DES CONSOMMATEURS DANS LE CONTEXTE \\ DU COMMERCE ÉLECTRONIQUE
}

\begin{abstract}
(C) OECD 2000
Genehmigungen zum Nachdruck von Teilen dieses Werks für nichtkommerzielle Zwecke oder zur Verwendung im Unterricht sind einzuholen beim Centre français d'exploitation du droit de copie (CFC), 20, rue des Grands-Augustins, 75006 Paris, Frankreich, Tel: (33-1) 44074770 , Fax: (33-1) 463467 19. Dies gilt für alle Länder mit Ausnahme der Vereinigten Staaten, wo das Copyright Clearance Center Inc. (CCC), Customer Service, Tel: (508)750-8400, 222 Rosewood Drive, Danvers, MA 01923, USA oder CCC online: http://www.copyright.com/ die entsprechenden Genehmigungen erteilt. Alle sonstigen Anträge auf Überlassung von Nachdruck- oder Übersetzungsrechten für das gesamte Dokument oder Teile davon sind zu richten an: OECD Publications, 2, rue André-Pascal, 75775 Paris Cedex 16, Frankreich.
\end{abstract}




\section{VORWORT}

Die Leitlinien für den Verbraucherschutz im Zusammenhang mit dem elektronischen Geschäftsverkehr, die am 9. Dezember 1999 vom Rat der OECD angenommen wurden, zielen darauf ab, Verbrauchern bei Online-Käufen zumindest einen ebenso hohen Schutz zu gewährleisten wie bei Einkäufen in herkömmlichen Geschäften oder bei Bestellungen im Versandhandel. Mit der Definition der wesentlichen Merkmale eines effektiven Verbraucherschutzes für elektronische Business-to-Consumer-Transaktionen sollen die Leitlinien einige der Unsicherheiten beseitigen helfen, mit denen Online-Käufe und -Verkäufe für Verbraucher und Unternehmen verbunden sind. Diese Leitlinien, die das Ergebnis 18-monatiger Diskussionen zwischen Regierungsvertretern aus OECD-Ländern sowie Repräsentanten von Wirtschafts- und Verbraucherverbänden darstellen, werden für die Regierungen, die Unternehmen und die Verbrauchervertreter eine wichtige Stütze dabei sein, Verbraucherschutzmechanismen für Online-Transaktionen zu entwickeln und umzusetzen, ohne dass dadurch Handelshemmnisse aufgerichtet werden.

Die Leitlinien orientieren sich an den in traditionelleren Handelsformen bereits existierenden gesetzlichen Verbraucherschutzmaßnahmen. Sie sollen folgende Ziele fördern: faire Geschäfts-, Werbe- und Marketingpraktiken; klare Informationen über die Identität von Online-Unternehmen, die angebotenen Waren oder Dienstleistungen sowie die Modalitäten und Bedingungen einer jeden Transaktion; transparente Verfahren für die Bestätigung von Transaktionen; sichere Zahlungsmechanismen; faire, zügige und finanziell tragbare Streitbeilegungs- und Abhilfeverfahren; Schutz der Privatsphäre und Aufklärung von Verbrauchern und Unternehmen. Sie sind technologieneutral, regen zu Initiativen des privaten Sektors unter Beteiligung von Verbrauchervertretern an und betonen die Notwendigkeit einer Zusammenarbeit zwischen Regierungen, Unternehmen und Verbrauchern.

Die Leitlinien wurden vom Ausschuss für Verbraucherpolitik unter dem Vorsitz von Jytte Ølgaard (Dänemark) verfasst und dem Rat der OECD vorgelegt. Der Text wurde mit Unterstützung einer Arbeitsgruppe erstellt, die mehrmals unter dem Vorsitz des Industry Canada's Office of Consumer Affairs tagte. Die späteren Fassungen der Leitlinien wurden von Laurie Labuda, Beraterin des OECD-Sekretariats, redigiert.

Für die Veröffentlichung des Berichts zeichnet der Generalsekretär der OECD verantwortlich. 


\section{INHALTSVERZEICHNIS}

Leitlinien für den Verbraucherschutz im Zusammenhang mit dem elektronischen Geschäftsverkehr

LEITLINIEN

TEIL I: GELTUNGSBEREICH _..............................................................

TEIL II: ALLGEMEINE GRUNDSÄTZE …………………………….......

I. TRANSPARENTER UND EFFEKTIVER SCHUTZ ............................ 9

II. FAIRE GESCHÄFTS-, WERBE- UND MARKETINGPRAKTIKEN. 10

III. ONLINE-INFORMATIONEN ........................................................... 11

A. INFORMATIONEN ÜBER DAS UNTERNEHMEN ........................ 11

B. INFORMATIONEN ÜBER DIE WAREN ODER DIENSTLEISTUNGEN ……......................................................... 12

C. INFORMATIONEN ÜBER DIE TRANSAKTION............................ 12

IV. BESTÄTIGUNGSVERFAHREN .................................................... 13

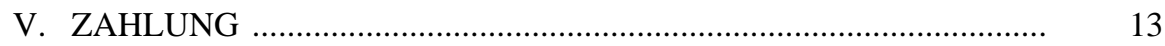

VI. STREITBEILEGUNG UND ABHILFE ………………………........... 14

A. ANWENDBARES RECHT UND ZUSTÄNDIGKEIT DER GERICHTE …............................................................. 14

B. ALTERNATIVE STREITBEILEGUNGS- UND ABHILFEVERFAHREN ..........................................................

VII. SCHUTZ DER PRIVATSPHÄRE ………………….......................... 15

VIII. AUFKLÄRUNG UND BEWUSSTSEINSBILDUNG …………........... 15

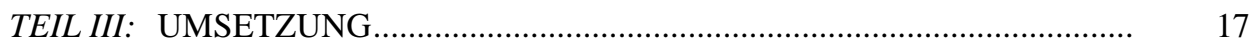

TEIL IV: GLOBALE ZUSAMMENARBEIT ……………………………....... 18 


\section{LEITLINIEN FÜR DEN VERBRAUCHERSCHUTZ IM ZUSAMMENHANG MIT DEM ELEKTRONISCHEN GESCHÄFTSVERKEHR}

Verbraucherschutzgesetze, -maßnahmen und -praktiken sorgen dafür, dass betrügerische, irreführende und unlautere Geschäftspraktiken in Grenzen gehalten werden. Derartige Schutzmaßnahmen sind unerlässlich, um das Vertrauen der Verbraucher zu gewinnen und ein ausgewogeneres Verhältnis zwischen Unternehmen und Verbrauchern bei kommerziellen Transaktionen zu schaffen.

Angesichts des naturgemäß internationalen Charakters digitaler Netzwerke und Computertechnologien, die den elektronischen Marktplatz bilden, ist es notwendig, dass auch für den Verbraucherschutz ein globaler Ansatz gewählt wird, und zwar als Element eines transparenten und vorhersehbaren Rechts- und Selbstregulierungsrahmens für den elektronischen Geschäftsverkehr. Unter den Bedingungen globaler Netzwerke werden die Kapazitäten jedes Landes bzw. jeder Gerichtsbarkeit auf die Probe gestellt, Probleme des Verbraucherschutzes im elektronischen Geschäftsverkehr angemessen zu lösen. Divergierende nationale Politiken könnten das Wachstum des elektronischen Geschäftsverkehrs behindern, und deshalb lassen sich diese Verbraucherschutzprobleme am effektivsten im Wege internationaler Konsultationen und Zusammenarbeit regeln. Die Regierungen der OECD-Mitgliedsländer sind sich der Tatsache bewusst, dass international koordinierte Ansätze für den Informationsaustausch und für eine generelle Verständigung über die Art und Weise, wie an diese Fragen heranzugehen ist, notwendig sein dürften.

Die Regierungen stehen vor der Herausforderung, auf der Grundlage der neu entstehenden Netzwerktechnologien die soziale Entwicklung und das Wirtschaftswachstum zu fördern und zugleich den Bürgern ihrer Länder einen effektiven und transparenten Verbraucherschutz im elektronischen Geschäftsverkehr zu bieten. Es gibt ein breites Spektrum von Verbraucherschutzgesetzen, in denen die Geschäftspraktiken geregelt sind. Viele OECD-Länder haben damit begonnen, ihre Verbraucherschutzgesetze und -praktiken unter dem Gesichtspunkt zu überprüfen, ob zur Integration der besonderen Aspekte des elektronischen Geschäftsverkehrs Änderungen vorgenommen werden müssen. Die Mitgliedsländer prüfen derzeit auch, in welcher Weise Selbstregulierungsmaßnahmen hier zu einem wirksamen und fairen Verbraucherschutz beitragen können. Zur Erreichung dieser Ziele sind Informationen und Beiträge aus breiten Kreisen der Zivilgesellschaft notwendig, und alle diese Initiativen sollten im Rahmen einer 
globalen Gemeinschaftsaktion von Regierungen, Unternehmen, Verbrauchern und deren Vertretern ergriffen werden.

Im April 1998 begann der OECD-Ausschuss für Verbraucherpolitik mit der Entwicklung eines Katalogs allgemeiner Leitlinien, die dem Schutz der am elektronischen Geschäftsverkehr teilnehmenden Verbraucher dienen, aber keine Handelshemmnisse aufrichten sollen. Diese Leitlinien stellen eine an Regierungen, Unternehmen, Verbraucher und deren Vertreter adressierte Empfehlung dar, die die wichtigsten Merkmale eines effektiven Verbraucherschutzes im elektronischen Geschäftsverkehr aufzeigt. Indessen soll keine der hierin enthaltenen Bestimmungen die eine oder andere Partei davon abhalten, über diese Leitlinien hinauszugehen, und die Mitgliedsländer auch nicht daran hindern, an strengeren Bestimmungen für den Online-Verbraucherschutz festzuhalten oder solche einzuführen. Der Zweck dieser Leitlinien besteht vor allem darin, mit der Vorgabe eines Rahmens sowie eines Katalogs von Grundsätzen Hilfe zu bieten für:

a) die Regierungen bei der Überprüfung, Formulierung und Umsetzung von Maßnahmen, Praktiken und gegebenenfalls Regulierungen zum Schutz des Verbrauchers sowie der Durchsetzung der einschlägigen Bestimmungen, um einen effektiven Verbraucherschutz im elektronischen Geschäftsverkehr zu gewährleisten;

b) die Unternehmensverbände, Verbrauchervereinigungen und Selbstregulierungsorgane durch Aufklärung über die wichtigsten Merkmale eines effektiven Verbraucherschutzes, die es bei der Überprüfung, Formulierung und Umsetzung von Selbstregulierungsprogrammen im elektronischen Geschäftsverkehr zu beachten gilt;

c) einzelne Unternehmen und Verbraucher, die sich am elektronischen Geschäftsverkehr beteiligen und denen hiermit klare Orientierungen bezüglich der Kernmerkmale gegeben werden, die Unternehmen im Hinblick auf die Offenlegung von Informationen und faire Geschäftspraktiken im elektronischen Geschäftsverkehr beachten müssen und die die Verbraucher erwarten können.

\section{DER RAT,}

In Anbetracht der vorstehenden Ausführungen und

Im Hinblick auf Artikel 5b des Übereinkommens über die Organisation für wirtschaftliche Zusammenarbeit und Entwicklung vom 14. Dezember 1960;

Im Hinblick auf die Erklärung der Minister über den Verbraucherschutz im Zusammenhang mit dem elektronischen Geschäftsverkehr vom 8./9. Oktober 1998 [C(98)177(Annex 2)]; 
Im Hinblick auf die Empfehlung des Rats zu den Leitlinien für den Schutz der Privatsphäre und den grenzüberschreitenden Verkehr personenbezogener Daten vom 23. September 1980 [C(80)58(Final)] sowie die Erklärung der Minister über den Schutz der Privatsphäre in globalen Netzen vom 8./9. Oktober 1998 [C(98)177(Annex 1)];

Im Hinblick auf die Erklärung der Minister zur Authentifizierung für den elektronischen Geschäftsverkehr vom 8./9. Oktober 1998 [C(98)177(Annex 3)];

Im Hinblick auf die Empfehlung des Rats zu den Leitlinien für die Sicherheit von Informationssystemen vom 26./27. November 1992 [C(92)188/FINAL] und die OECD-Empfehlung zu den Leitlinien für die Kryptographiepolitik vom 27. März 1997 [C(97)62/FINAL];

In der Erkenntnis, dass der elektronische Geschäftsverkehr Verbrauchern neue und wesentliche Vorteile bieten kann, namentlich mehr Komfort, Zugang zu einer breiten Palette von Waren und Dienstleistungen sowie die Möglichkeit, Informationen über diese Waren und Dienstleistungen zusammenzutragen und zu vergleichen;

In der Erkenntnis, dass gewisse spezifische Merkmale des elektronischen Geschäftsverkehrs, wie die Einfachheit und Geschwindigkeit, mit denen Unternehmen und Verbraucher über die Waren und Dienstleistungen kommunizieren und grenzüberschreitende Transaktionen abwickeln können, möglicherweise zu Geschäftssituationen führen, die den Verbrauchern nicht vertraut sind und die ihre Interessen gefährden könnten, und es für Verbraucher und Unternehmen deshalb zunehmend wichtig ist, über ihre Rechte und Pflichten am elektronischen Marktplatz informiert und sich dieser bewusst zu sein;

In der Erkenntnis, dass die Regeln zum Verbraucherschutz für das anwendbare Recht und die Zuständigkeit der Gerichte Auswirkungen auf zahlreiche Aspekte des elektronischen Geschäftsverkehr haben können, ebenso wie die Regeln betreffend das anwendbare Recht und die Zuständigkeit der Gerichte in anderen Bereichen möglicherweise den Verbraucherschutz beeinflussen;

In der Erkenntnis, dass das Verbrauchervertrauen in den elektronischen Geschäftsverkehr gestärkt wird durch die kontinuierliche Entwicklung transparenter und effektiver Verbraucherschutzmechanismen, die betrügerische, irreführende oder unlautere Geschäftspraktiken im Online-Handel begrenzen;

In der Erwägung, dass der elektronische Geschäftsverkehr allen Verbrauchern offen stehen und zugänglich sein sollte; und

In der Erwägung, dass Regierungen, Unternehmen, Verbraucher und deren Vertreter der Entwicklung effizienter grenzüberschreitender Abhilfeverfahren besondere Aufmerksamkeit widmen sollten. 


\section{EMPFIEHLT DEN MITGLIEDSLÄNDERN:}

Die notwendigen Maßnahmen zur Umsetzung der einschlägigen Bestimmungen der im Anhang zu dieser Empfehlung enthaltenen Leitlinien zu ergreifen;

Für eine weite Verbreitung der Leitlinien in allen zuständigen Ministerien und Behörden, den am elektronischen Geschäftsverkehr beteiligten Unternehmen, bei Verbrauchervertretern, in den Medien, Bildungseinrichtungen und sonstigen relevanten öffentlichen Interessengruppen zu sorgen;

Unternehmen, Verbraucher und ihre Vertreter zur Übernahme einer aktiven Rolle bei der Förderung der Umsetzung der Leitlinien auf internationaler, nationaler und lokaler Ebene zu ermutigen;

Regierungen, Unternehmen, Verbraucher und ihre Vertreter zur Mitwirkung bei der Ausarbeitung und Beurteilung von Empfehlungen zu der laufenden Überprüfung der Regeln betreffend das anwendbare Recht und die Zuständigkeit der Gerichte zu ermutigen;

Nichtmitgliedsländer zu ersuchen, bei der Überprüfung ihrer Verbraucherschutzmaßnahmen und entsprechender Initiativen und Regulierungen dem Inhalt dieser Empfehlung Rechnung zu tragen;

Konsultationen in die Wege zu leiten, zusammenzuarbeiten und den Informationsaustausch untereinander und mit Nichtmitgliedsländern, Unternehmen, Verbrauchern und deren Vertretern auf nationaler wie auch auf internationaler Ebene zu erleichtern, um gemäß den Leitlinien einen effektiven Verbraucherschutz im elektronischen Geschäftsverkehr zu bieten;

Die Leitlinien in einer Weise umzusetzen, die der Entwicklung neuer Unternehmensmodelle und technologischer Anwendungen, die den Verbrauchern zugute kommen, förderlich ist; und die Verbraucher dazu zu ermutigen, alle zur Stärkung ihrer Position als Käufer verfügbaren Instrumente zu nutzen; und

BEAUFTRAGT den Ausschuss für Verbraucherpolitik damit, Informationen über Fortschritte und Erfahrungen bei der Umsetzung der vorliegenden Empfehlung auszutauschen, diese Informationen zu überprüfen und dem Rat im Jahr 2002 oder früher und gegebenenfalls auch in der Folgezeit hierüber Bericht $\mathrm{zu}$ erstatten. 


\section{LEITLINIEN}

\section{TEIL I}

\section{GELTUNGSBEREICH}

Diese Leitlinien gelten nur für den elektronischen Geschäftsverkehr im Business-to-Consumer-Verhältnis und nicht für Business-to-Business-Transaktionen.

\section{TEIL II}

\section{ALLGEMEINE GRUNDSÄTZE}

\section{TRANSPARENTER UND EFFEKTIVER SCHUTZ}

Verbraucher, die am elektronischen Geschäftsverkehr teilnehmen, sollten einen transparenten und effektiven Verbraucherschutz genießen, der zumindest dem in anderen Geschäftsformen garantierten Niveau entspricht.

Regierungen, Unternehmen, Verbraucher und deren Vertreter sollten zusammenarbeiten, um ein entsprechendes Schutzniveau zu erreichen und zu entscheiden, welche Änderungen zur Berücksichtigung der spezifischen Merkmale des elektronischen Geschäftsverkehrs eventuell vorgenommen werden müssen. 


\section{FAIRE GESCHÄFTS-, WERBE- UND MARKETINGPRAKTIKEN}

Die Unternehmen, die elektronischen Geschäftsverkehr betreiben, sollten den Interessen der Verbraucher gebührend Rechnung tragen und faire Geschäfts-, Werbe- und Marketingpraktiken anwenden.

Die Unternehmen sollten sich aller Darstellungen enthalten bzw. alle Auslassungen vermeiden und keine Praktiken anwenden, die täuschend, irreführend, betrügerisch oder unfair sein könnten.

Die Unternehmen, die dem Verbraucher Waren oder Dienstleistungen zum Verkauf anbieten, dafür werben oder diese vermarkten, sollten keine Praktiken anwenden, die mit einem ungebührlichen Schadensrisiko für den Verbraucher verbunden sind.

Wenn Unternehmen Informationen über sich selbst bzw. ihr Waren- oder Dienstleistungsangebot bereitstellen, sollten sie diese Informationen in klarer, deutlich sichtbarer, sachlich richtiger und leicht zugänglicher Art und Weise präsentieren.

Das Geschäftsverhalten der Unternehmen sollte mit allen Darstellungen von Maßnahmen oder Praktiken übereinstimmen, die sie im Hinblick auf ihre Transaktionen mit Verbrauchern anwenden.

Die Unternehmen sollten dem globalen Charakter des elektronischen Geschäftsverkehrs Rechnung tragen und soweit möglich die unterschiedlichen Regelungsmerkmale ihrer Zielmärkte berücksichtigen.

Die Unternehmen sollten die spezifischen Merkmale des elektronischen Geschäftsverkehrs nicht dazu nutzen, ihre wahre Identität oder ihren Standort zu verschleiern oder die Einhaltung von Verbraucherschutzvorschriften und/oder Durchsetzungsmechanismen zu umgehen.

Die Unternehmen sollten keine unfairen Vertragsklauseln anwenden.

Werbung und Marketing sollten als solche klar erkennbar sein.

In der Werbung und beim Marketing sollte die Identität des Unternehmens, für das die Werbung bzw. das Marketing durchgeführt wird, angegeben werden, wenn die Unterlassung dieser Angaben einer Irreführung gleichkäme.

Die Unternehmen sollten in der Lage sein, für die Korrektheit aller expliziten oder impliziten Äußerungen, solange diese aufrechterhalten werden - und auch noch für eine angemessene Zeit danach -, den Nachweis zu erbringen. 
Die Unternehmen sollten effiziente und benutzerfreundliche Verfahren entwickeln und umsetzen, die den Verbrauchern die Entscheidung darüber überlassen, ob sie unaufgeforderte Werbung per E-mail bekommen wollen oder nicht.

Haben die Verbraucher angegeben, den Empfang unaufgeforderter Werbung per E-mail nicht zu wünschen, sollte ihre Wahl respektiert werden.

In einer Reihe von Ländern gelten für die unaufgeforderte Werbung per E-mail spezifische Gesetzes- bzw. Selbstregulierungsanforderungen.

Die Unternehmen sollten bei der Gestaltung von Werbung und Marketing für Zielgruppen wie Kinder, ältere Menschen, Schwerkranke und andere Personen, die u.U. nicht in der Lage sind, die präsentierten Informationen voll zu verstehen, besondere Sorgfalt üben.

\section{ONLINE-INFORMATIONEN}

\section{A. INFORMATIONEN ÜBER DAS UNTERNEHMEN}

Unternehmen, die mit Verbrauchern elektronischen Geschäftsverkehr betreiben, sollten exakte, klare und leicht zugängliche Informationen über sich selbst liefern, und zwar in hinreichendem Umfang, die mindestens ermöglichen:

a) Die Identifizierung des Unternehmens - einschließlich seiner Rechtsform und der Firmenbezeichnung, der geographischen Adresse des Unternehmenssitzes, der E-mail-Adresse oder anderer elektronischer Kontaktmöglichkeiten bzw. Telefonnummern sowie gegebenenfalls einer Adresse für Registrierungszwecke und etwaiger amtlicher Registrier- oder Lizenznummern.

b) Schnelle, einfache und effektive Kommunikation zwischen Verbraucher und Unternehmen.

c) Geeignete und effiziente Streitbeilegung.

d) Zustellung von amtlichen Schriftstücken.

e) Feststellung des Unternehmenssitzes und der Geschäftsführer des Unternehmens durch Gesetzesvollzugs- und Regulierungsbehörden.

Gibt ein Unternehmen seine Zugehörigkeit zu einem Selbstregulierungsmechanismus, einem Unternehmensverband, einer Streitbeilegungseinrichtung oder einer anderen Zertifizierungsstelle an, sollte es dem Verbraucher entsprechende Kontaktstellen sowie eine einfache Methode nennen, mit der er diese Zugehörigkeit überprüfen und sich Zugang zu den einschlägigen Normen und Praktiken der Zertifizierungsstelle verschaffen kann. 


\section{B. INFORMATIONEN ÜBER DIE WAREN ODER DIENSTLEISTUNGEN}

Unternehmen, die mit Verbrauchern elektronischen Geschäftsverkehr betreiben, sollten exakte und leicht zugängliche Informationen zur Beschreibung ihres Waren- oder Dienstleistungsangebots bereitstellen, und zwar in hinreichendem Umfang, damit die Verbraucher eine verständige Entscheidung über die Durchführung einer Transaktion treffen können, und in einer Form, die es den Verbrauchern ermöglicht, eine sachdienliche Aufzeichnung der Informationen zu erhalten.

\section{INFORMATIONEN ÜBER DIE TRANSAKTION}

Unternehmen, die elektronischen Geschäftsverkehr betreiben, sollten hinreichende Informationen über die Modalitäten, die Bedingungen und die mit einer Transaktion verbundenen Kosten liefern, damit die Verbraucher eine verständige Entscheidung darüber treffen können, ob sie eine Transaktion durchführen wollen oder nicht.

Diese Informationen sollten klar, exakt, leicht zugänglich sein und in einer Weise bereitgestellt werden, die den Verbrauchern hinreichende Möglichkeiten gibt, sie vor Durchführung der Transaktion zu überprüfen.

Wenn die Transaktion in mehreren Sprachen durchgeführt werden kann, sollten die Unternehmen alle Informationen, die die Verbraucher benötigen, um eine verständige Entscheidung über die Transaktion treffen zu können, in den betreffenden Sprachen bereitstellen.

Die Unternehmen sollten den Verbrauchern einen klar verständlichen und vollständigen Text der für die Transaktionen geltenden Geschäftsbedingungen liefern, und zwar so, dass sich die Verbraucher Zugang zu diesen Informationen verschaffen und davon eine sachdienliche Aufzeichnung erhalten können.

Je nach Transaktion sollten diese Informationen, soweit notwendig und angemessen, folgende Elemente beinhalten:

a) Eine genaue Aufstellung aller vom Unternehmen eingezogenen und/ oder von diesem berechneten Kosten.

b) Hinweis auf andere zusätzliche Kosten, die normalerweise vom Verbraucher zu tragen sind und die nicht vom Unternehmen eingezogen und/oder berechnet werden.

c) Liefer- und Vertragserfüllungsbedingungen. 
d) Zahlungsbedingungen, -modalitäten und -weisen.

e) Beim Kauf zu beachtende Vorbehalte, Beschränkungen oder Auflagen, wie Zustimmungsbedürftigkeit der Eltern oder des Erziehungsberechtigten, geographische oder zeitliche Beschränkungen.

f) Gebrauchsanweisungen mit Sicherheitshinweisen und Warnungen vor Gesundheitsrisiken.

g) Informationen über den verfügbaren Kundendienst.

h) Einzelheiten und Modalitäten bezüglich Widerruf, Kündigung, Rückgabe, Umtausch, Stornierung und/oder Geldrückerstattung.

i) Bestimmungen über Gewährleistungen und Garantien.

Bei allen Informationen über Kosten sollte die verwendete Währung angegeben werden.

\section{BESTÄTIGUNGSVERFAHREN}

Um allen Missverständnissen in Bezug auf die Kaufabsichten des Verbrauchers vorzubeugen, sollte dieser in der Lage sein, vor Abgabe seiner Bestellung die Waren oder Dienstleistungen, die er zu kaufen wünscht, genau zu identifizieren, etwaige Fehler zu erkennen und zu korrigieren oder die Bestellung zu ändern, dem Kaufangebot verständig und bewusst zuzustimmen und eine vollständige und exakte Aufzeichnung der Transaktion zu erhalten .

Es sollte für den Verbraucher möglich sein, die Transaktion vor Abgabe seiner Bestellung zu beenden.

\section{ZAHLUNG}

Den Verbrauchern sollten einfache, benutzerfreundliche und sichere Zahlungsmechanismen sowie Informationen über den Sicherheitsgrad der angebotenen Mechanismen zur Verfügung gestellt werden.

Haftungsbeschränkungen bei unbefugter oder betrügerischer Benutzung von Zahlungssystemen und Rückerstattungsmechanismen sind sehr wirksame Instrumente zur Stärkung des Verbrauchervertrauens; im Zusammenhang mit dem elektronischen Geschäftsverkehr sollten sie deshalb ausgebaut und ihre Anwendung gefördert werden. 


\section{STREITBEILEGUNG UND ABHILFE}

\section{A. ANWENDBARES RECHT UND ZUSTÄNDIGKEIT DER GERICHTE}

Grenzüberschreitende Business-to-Consumer-Transaktionen, ob sie nun elektronisch oder auf anderem Wege abgewickelt werden, unterliegen dem bestehenden Rahmen für die Regeln betreffend das anwendbare Recht und die Zuständigkeit der Gerichte.

Der elektronische Geschäftsverkehr stellt diese bestehenden Rahmenbedingungen vor neue Herausforderungen. Daher müssen Überlegungen angestellt werden, ob der existierende Rahmen für die Regeln betreffend das anwendbare Recht und die Zuständigkeit der Gerichte geändert oder anders angewendet werden sollte, um einen effektiven und transparenten Verbraucherschutz im kontinuierlich wachsenden elektronischen Geschäftsverkehr zu gewährleisten.

Bei Überlegungen hinsichtlich einer Anpassung des bestehenden Rechtsrahmens sollten die Regierungen bestrebt sein sicherzustellen, dass dieser faire Bedingungen für Verbraucher und Unternehmen vorsieht, die den elektronischen Geschäftsverkehr erleichtern, den Verbrauchern einen zumindest ebenso hohen Schutz garantieren wie bei anderen Geschäftsformen und ihnen effektiven $\mathrm{Zu}$ gang zu fairen und zügigen Streitbeilegungs- bzw. Abhilfeverfahren sichern, die keine ungebührlichen Kosten oder Belastungen verursachen.

\section{B. ALTERNATIVE STREITBEILEGUNGS- UND ABHILFEVERFAHREN}

Die Verbraucher sollten effektiven Zugang erhalten zu fairen und zügigen alternativen Streitbeilegungs- und Abhilfeverfahren, die keine ungebührlichen Kosten oder Belastungen verursachen.

Unternehmen, Verbrauchervertreter und Regierungen sollten gemeinsam darauf hinarbeiten, weiterhin faire, wirksame und transparente Selbstregulierungsmechanismen sowie andere Maßnahmen und Verfahren anzuwenden und zu entwickeln, darunter alternative Streitbeilegungsmechanismen für den Umgang mit Verbraucherbeschwerden und die Beilegung von Streitigkeiten mit Verbrauchern, die durch den elektronischen Geschäftsverkehr im Business-to-Consumer-Verhältnis hervorgerufen werden, wobei grenzüberschreitenden Transaktionen besondere Aufmerksamkeit gebührt:

a) Unternehmen und Verbrauchervertreter sollten weiterhin faire, wirksame und transparente interne Mechanismen für die Behandlung von Verbraucherbeschwerden und anderen Problemen einrichten, damit diese Fälle in fairer und zügiger Weise gelöst werden, ohne dass unge- 
bührliche Kosten oder Belastungen für den Verbraucher entstehen. Die Verbraucher sollten zur Inanspruchnahme dieser Mechanismen ermutigt werden.

b) Unternehmen und Verbrauchervertreter sollten weiterhin gemeinsame Selbstregulierungsprogramme für die Behandlung von Verbraucherbeschwerden und die Unterstützung der Verbraucher bei der Beilegung von Streitigkeiten einrichten, die durch den elektronischen Geschäftsverkehr auf Business-to-Consumer-Ebene hervorgerufen werden.

c) Unternehmen, Verbrauchervertreter und Regierungen sollten gemeinsam darauf hinarbeiten, den Verbrauchern weiterhin die Wahl alternativer Streitbeilegungsmechanismen bieten zu können, die es ermöglichen, den Streitfall in fairer und zügiger Weise effektiv beizulegen, ohne dass ungebührliche Kosten oder Belastungen für den Verbraucher entstehen.

d) Bei der Umsetzung der vorstehenden Punkte sollten die Unternehmen, Verbrauchervertreter und Regierungen Informationstechnologien innovativ einsetzen und dazu nutzen, die Verbraucher besser aufzuklären und ihnen größere Wahlmöglichkeiten zu geben.

Darüber hinaus bedarf es zusätzlicher Untersuchungen, um die in Abschnitt VI dargelegten Ziele auf internationaler Ebene zu erreichen.

\section{SCHUTZ DER PRIVATSPHÄRE}

Der elektronische Geschäftsverkehr auf Business-to-Consumer-Ebene sollte im Einklang mit den anerkannten und in den OECD-Leitlinien für den Schutz der Privatsphäre und den grenzüberschreitenden Verkehr personenbezogener Daten (1980) dargelegten Grundsätzen zum Schutz der Privatsphäre wie auch unter Berücksichtigung der OECD-Ministererklärung über den Schutz der Privatsphäre in globalen Netzen (1998) betrieben werden, um Verbrauchern einen angemessenen und effektiven Schutz zu gewährleisten.

\section{AUFKLÄRUNG UND BEWUSSTSEINSBILDUNG}

Regierungen, Unternehmen und Verbrauchervertreter sollten zusammenarbeiten, um die Verbraucher über spezifische Merkmale des elektronischen Geschäftsverkehrs aufzuklären, dafür zu sorgen, dass die am elektronischen Geschäftsverkehr teilnehmenden Verbraucher verständige Entscheidungen treffen können, und das Bewusstsein von Unternehmen und Verbrauchern hinsichtlich der Verbraucherschutzbestimmungen, die für ihre Online-Aktivitäten gelten, zu schärfen. 
Regierungen, Unternehmen, Medien, Bildungseinrichtungen und Verbrauchervertreter sollten alle wirksamen Mittel zur Aufklärung von Verbrauchern und Unternehmen nutzen, namentlich die durch die globalen Netze möglich gemachten innovativen Techniken.

Regierungen, Verbrauchervertreter und Unternehmen sollten gemeinsam darauf hinarbeiten, Verbraucher und Unternehmen weltweit auf leicht zugängliche und verständliche Art und Weise über relevante Verbraucherschutzgesetze und Rechtsschutzinstrumente zu informieren.

\section{TEIL III}

\section{UMSETZUNG}

Damit diese Empfehlung ihren Zweck erfüllt, sollten die Mitgliedsländer auf nationaler und internationaler Ebene und in Zusammenarbeit mit Unternehmen, Verbrauchern und ihren Vertretern:

a) Selbstregulierungspraktiken überprüfen und gegebenenfalls fördern und/oder Gesetze bzw. Praktiken einführen oder dahingehend ändern, dass sie auf den elektronischen Geschäftsverkehr anwendbar sind, wobei die Grundsätze der Technologie- und Medienneutralität beachtet werden müssen.

b) Den privaten Sektor ermutigen, weiterhin eine Führungsrolle zu übernehmen, und zwar unter Beteiligung von Verbrauchervertretern an der Entwicklung wirksamer Selbstregulierungsmechanismen, die spezifische Grundregeln für Streitbeilegungs- und Konformitätsmechanismen beinhalten.

c) Den privaten Sektor ermutigen, in der Technologieentwicklung als Instrument zum Schutz und zur Stärkung der Position des Verbrauchers weiterhin eine Führungsrolle zu übernehmen.

d) Die Existenz, den Zweck und den Inhalt der Leitlinien einem möglichst breiten Publikum nahe bringen und deren Anwendung fördern.

e) Den Verbrauchern sowohl den Zugang zu Informations- und Beratungsquellen über Verbraucherschutz als auch die Einlegung von Beschwerden im Zusammenhang mit dem elektronischen Geschäftsverkehr erleichtern. 


\section{TEIL IV}

\section{GLOBALE ZUSAMMENARBEIT}

Um einen effektiven Verbraucherschutz im Zusammenhang mit dem globalen elektronischen Geschäftsverkehr zu gewährleisten, sollten die Mitgliedsländer:

a) Die Kommunikation, die Zusammenarbeit und gegebenenfalls die Entwicklung und Durchsetzung gemeinsamer Initiativen auf internationaler Ebene zwischen Unternehmen, Verbrauchervertretern und Regierungen erleichtern.

b) Durch ihre Justiz-, Regulierungs- und Vollzugsbehörden auf internationaler Ebene zusammenarbeiten und - entsprechend der jeweiligen Situation - durch Informationsaustausch, Koordinierung, Kommunikation und gemeinsames Vorgehen zur Bekämpfung grenzüberschreitender betrügerischer, irreführender und unfairer Geschäftspraktiken beitragen.

c) Die existierenden internationalen Netze nutzen und, soweit notwendig und angemessen, zur Verwirklichung einer solchen Zusammenarbeit bilaterale und/oder multilaterale Übereinkommen oder andere Vereinbarungen treffen.

d) Auf nationaler wie auf internationaler Ebene auf eine Konsensbildung in wesentlichen Fragen des Verbraucherschutzes hinarbeiten, um die angestrebten Ziele - Stärkung des Verbrauchervertrauens, Gewährleistung verlässlicher Rahmenbedingungen für Unternehmen und Schutz des Verbrauchers - zu fördern.

e) Durch Kooperationsbemühungen auf den Abschluss von Übereinkommen oder Vereinbarungen für die gegenseitige Anerkennung und Vollstreckung von Entscheidungen hinarbeiten, die in Streitfällen zwischen Verbrauchern und Unternehmen gefällt werden bzw. zur Durchsetzung von Maßnahmen zur Bekämpfung betrügerischer, irreführender oder unfairer Geschäftspraktiken ergehen. 
OECD PUBLICATIONS, 2, rue André-Pascal, 75775 PARIS CEDEX 16 PRINTED IN FRANCE

(93 2000025 P) ISBN 92-64-57636-3 - No. 513612000 